\title{
METABOLIC SYNDROME AMONG DOCTORS: A PILOT STUDY FROM ODISHA
}

\section{MAGNA MANJAREEKA ${ }^{1 *}$, SOUMYA MISHRA ${ }^{1}$, PRAKASH K NAYAK ${ }^{1}$, SHUBHRANSU PATRO ${ }^{2}$, JAYANTI MISHRA ${ }^{1}$, SURESH C DASH ${ }^{3}$}

${ }^{1}$ Department of Physiology, Kalinga Institute of Medical Sciences, KIIT University, Bhubaneswar, Odisha, India. ${ }^{2}$ Department of Medicine, Kalinga Institute of Medical Sciences, KIIT University, Bhubaneswar, Odisha, India. ${ }^{3}$ Department of Nephrology, Kalinga Institute of Medical Sciences, KIIT University, Bhubaneswar, Odisha, India. Email: dr.magna@gmail.com

Received: 17 May 2018, Revised and Accepted: 20 June 2018

\section{ABSTRACT}

Objective: This study was planned to assess the prevalence of metabolic syndrome (MetS) among doctors of Bhubaneswar.

Methods: A cross-sectional, single-centered observational study was conducted among doctors over a period of 8 months after clearance from Institutional Ethical Committee. All consented participants were subjected to anthropometric measurements and physical examination. Fasting plasma glucose and complete lipid profile were estimated using standard procedures after $12 \mathrm{~h}$ of overnight fast. MetS was defined according to the Modified National Cholesterol Education Program-Adult Treatment Panel III criteria for MetS screening.

Results: Among 170 participants (males - 117 and females - 53), 64 (37.65\%) were with MetS. Prevalence of same was higher in males (41.9\%) than females $(28.3 \%)$. The younger doctors ( $\leq 40$ years) had more prevalence of MetS compared to the elderly ( $>40$ years) doctors ( 41 [39.8\%] vs. 23 [34.3\%]). In the males, high blood pressure was the major contributor to the prevalence of MetS followed by low high-density lipoproteins-cholesterol (HDL-C) and high triglyceride values. However, in females, low HDL-C and greater waist circumference contributed the maximum to MetS.

Conclusion: In the present study, the young male doctors have a greater prevalence of MetS than the older and the females. MetS is still a significant public health problem in the educated population including those of doctors, especially the younger ones.

Keywords: Cardiovascular risk factors, Lipid profile, Health-care provider, Physician.

(C) 2018 The Authors. Published by Innovare Academic Sciences Pvt Ltd. This is an open access article under the CC BY license (http://creativecommons. org/licenses/by/4. 0/) DOI: http://dx.doi.org/10.22159/ajpcr.2018.v11i10.27330

\section{INTRODUCTION}

Metabolic syndrome (MetS) is described as clustering of several risk factors for cardiovascular diseases (CVD) such as hypertension, dyslipidemia, central obesity, insulin resistance, and high fasting plasma glucose (FPG) [1]. The former in turn may give rise to a number of secondary complications [2]. Prevalence of MetS is known to be significantly increased in the Asian countries [3]. A study conducted in urban areas of Karachi, Pakistan, showed a high prevalence of the MetS (49\%) [4]. A recent multi-ethnic population-based survey reports the highest prevalence of MetS in Indian ethnicity [5]. One-third of the urban population in large cities in India has MetS [6]. Recent community-based studies from eastern India, West Bengal, and Odisha, have reported the prevalence of MetS to be $31.4 \%$ and $43.2 \%$, respectively $[7,8]$.

Individual lifestyle particularly psychosocial or work stress, lack of regular physical activity, and smoking are strongly associated with obesity and MetS $[9,10]$. Exposure to job-specific stress increases the prevalence of MetS [11,12]. A recent study reported prevalence of stress to be very high in resident doctors in Delhi [13]. Since doctors are considered to be representing a highly educated mass of the population, their awareness and knowledge regarding the health consequences of lifestyle changes are generally expected to be high. Although adequate information is available on such lifestyle disorders among the general population from different parts of India, the information regarding the same is scarcely available from doctors of India $[14,15]$. With such a background in mind, this study plans to assess the prevalence of MetS among the doctors of Bhubaneswar, Odisha, in the eastern part of India.

\section{METHODS}

\section{Study design}

A cross-sectional, single-center observational study was conducted at a tertiary care hospital and medical college over a period of
8 months after obtaining permission from the Institutional Ethical Committee.

\section{Sample size estimation}

The sample size for this study was determined using the prevalence of MetS among the medical community of a tertiary health center in Pakistan where the researchers have found the prevalence to be $14.95 \%$ [16]. This study was selected for sample size calculation as the Indian studies where the prevalence of health professionals has been done were very old. The sample size for prevalence study was calculated using the formula $\mathrm{n}=\mathrm{Z}^{2} \mathrm{pq} / \mathrm{d}^{2}$, where $\mathrm{n}$ is the desired sample size, $\mathrm{z}$ is the standard estimate $=1.96$ at $5 \%$ level of significance, $p=$ prevalence of MetS among physicians, i.e., $14.9 \%, q=1-p$ and $d$ is the precision of the study $=0.06(6 \%)$. Using the formula, the sample size was estimated to be 142 .

\section{Data collection}

A total of 170 doctors aged between 24 and 65 years and working in KIMS, participated and were included for this study. Simple questionnaires pertaining to vegetarian or non-vegetarian diet, smoking and alcohol habits, exercise, prior medical history of drug intake, and any relevant family history of CVD, diabetes mellitus, hypertension or chronic kidney disease were answered by the doctors. Subjects with any coexisting serious diseases, those taking antidiabetic drugs/insulin, lipid-lowering drugs, and/or anti-hypertensive drugs were excluded from the study.

Of the 170 doctors included in the study 117 were males and 53 were females. All participants were subjected to anthropometric measurements such as height, weight, and waist circumference (WC) using standard procedures [17]. Body mass index (BMI) was computed using the formula: BMI=Weight (in $\mathrm{kg}$ )/(Height [in $\mathrm{m}$ ] $)^{2}$ [18]. Right arm blood pressure (BP) was measured by mercury sphygmomanometer in sitting position 
after 15 min of complete physical and mental relaxation. Three readings were recorded by palpatory and auscultatory methods by trained observers; the average of higher two readings by the latter method was considered in this study. Blood samples were taken after a $12 \mathrm{~h}$ overnight fast, and plasma was separated immediately by centrifugation. Fasting plasma glucose (FPG), triglycerides (TG), total cholesterol, high-density lipoproteins (HDL), low-density lipoproteins, and very low-density lipoproteins were estimated by standardized procedures followed in the Central Laboratory of the institute. Plasma glucose was estimated by glucose - oxidase-peroxidase method and lipids by standard enzymatic method using Hitachi 902 Auto-analyzer. Reagents of Roche Diagnostics (Germany) were used [19]. Subjects with FPG between 100 and $125 \mathrm{mg} /$ $\mathrm{dL}$ were diagnosed as impaired fasting glucose (IFG), while subjects having FPG equal to or $>126 \mathrm{mg} / \mathrm{dL}$ were diagnosed as diabetes mellitus (DM). MetS was defined according to Modified National Cholesterol Education Program-Adult Treatment Panel III (NCEP-ATP III) criteria [20]. Table 1 describes Modified NCEP ATPIII criteria for MetS screening. Presence of at least 3 of the 5 risk factors defines MetS in an individual.

\section{Statistical analysis}

Chi-square tests were performed to determine overall differences in the frequencies of categorical variables such as BMI, WC, BP, TGs, fasting blood sugar, and HDL-C among the gender groups and also age groups ( $\leq 40$ years and $>40$ years). $p<0.05$ was considered as statistically significant. All analyses were carried out using SPSS (Version 22).

\section{RESULTS}

All the 170 participants (117 males and 53 females) were classified into groups: (A) Those $\leq 40$ years of age, gender-wise and (B) those

\section{Table 1: Modified NCEP ATPIII criteria for metabolic syndrome} screening

\begin{tabular}{ll}
\hline Risk factors & Cutoff points \\
\hline WC & Males $>90 \mathrm{~cm}$, females $>80 \mathrm{~cm}$ \\
SBP/DBP & $\geq 130 \mathrm{~mm}$ of Hg and $/$ or $\geq 85 \mathrm{~mm}$ of $\mathrm{Hg}$ \\
TG & $\geq 150 \mathrm{mg} / \mathrm{dL}$ \\
HDL-C & $<40 \mathrm{mg} / \mathrm{dL}-$ males, $<50 \mathrm{mg} / \mathrm{dL}-$ females \\
Fasting plasma sugar & $\geq 100 \mathrm{mg} / \mathrm{dL}$
\end{tabular}

WC: Waist circumference, TG: Triglycerides, SBP: Systolic blood pressure

DBP: Diastolic blood pressure, HDL-C: High-density lipoprotein cholesterol

Table 2: The prevalence of MetS in each described groups

\begin{tabular}{lllll}
\hline Prevalence & \multicolumn{2}{l}{ Age $\leq$ 40 years } & \multicolumn{2}{l}{ Age $>$ 40 years } \\
\hline $\mathrm{N}$ & Males (72) & Females (31) & Males (45) & Females (22) \\
MetS & $31(43.1)$ & $10(32.3)$ & $18(40.0)$ & $05(22.7)$ \\
present (\%) & & & & \\
\hline
\end{tabular}

MetS: Metabolic syndrome
$>40$ years of age, again gender-wise. Of the 117 males, 49 (41.88\%) of them were screened to have MetS. Similarly, 15 (28.30\%), of 53 females had MetS. In both the genders, MetS was more prevalent (39.81\%) in the age group $\leq 40$ years than that of $>40$ years group (34.33\%) which was statistically insignificant $(\mathrm{p}=0.52)$ as shown in Table 4 .

Table 3 shows the gender differences of occurrence of various disorders. While males showed significantly higher percentage of increase in BP and increase in TG than the females, more percentage of females were seen having decreased HDL and increased WC. There was no significant difference in the prevalence of MetS and prevalence of DM in both the groups.

It is evident from Table 4 that there were not significant differences seen in the various disorders in both the age groups except for higher prevalence of increased BP and IFG in the elderly group and higher prevalence of increased TG and decreased HDL in the younger age group.

In the males, high DBP was the major contributor to the prevalence of MetS, which was followed by low HDL-cholesterol (HDL-C) and high TG values. However, in females, low HDL-C, and greater WC contributed maximum to MetS (Table 5).

\section{DISCUSSION}

Doctors are equally prone to lifestyle diseases like MetS as the general population. Foreign studies state that physicians maintain very good health habits compared to the general population and when these physicians explain self-practiced health habits to their patients it effectively stimulates the patients to follow the healthy habits $[21,22]$. However, the paucity of time for exercise, sedentary lifestyle, higher socioeconomic status and challenge of balancing personal, and professional lives are few of the many factors which could explain the lack of adequate health care of doctors in our country [23]. This study was undertaken to find out the magnitude of doctors having MetS in a tertiary care health institute.

In this study, the prevalence of MetS among doctors was $37.65 \%$. A similar prevalence (37.1\%) was seen in a study by Garg et al. [24]. Prevalence of MetS in the general population from different parts of India showed varied reports. Prevalence data of MetS observed in studies from the North [25,26], East, [7] West [27], and South India [28] were lower compared to this study. In contrast, results of a previous study show prevalence of MetS among doctors are more common than the general population which may be attributed to higher socioeconomic status, less physical activity and more stressful working atmosphere for the former sector of population [15]. In the present study, though we have not done a comparison with the general population, still results of a previous study conducted in urban healthy adults of Southern Odisha show a higher prevalence of MetS (43.2\%) than ours [8].

Table 3: Comparison of prevalence of various disorders among males and females

\begin{tabular}{|c|c|c|c|c|}
\hline Risk factors & Male (\%) & Female (\%) & Total (\%) & $p$ value \\
\hline $\mathrm{N}$ & $117(68.8)$ & $53(31.2)$ & $170(100)$ & \\
\hline $\mathrm{SBP} \geq 130 \mathrm{~mm} \mathrm{Hg}$ & $56(47.9)$ & $12(22.6)$ & $68(40.0)$ & $0.002^{*}$ \\
\hline $\mathrm{DBP} \geq 85 \mathrm{~mm} \mathrm{Hg}$ & $50(42.7)$ & $10(18.9)$ & $60(35.3)$ & $0.003^{*}$ \\
\hline $\mathrm{TG} \geq 150 \mathrm{mg} / \mathrm{dl}$ & $40(34.2)$ & $10(18.9)$ & $50(29.4)$ & $0.046^{*}$ \\
\hline HDL $<$ cut off ${ }^{\dagger}$ & $39(33.3)$ & $27(50.9)$ & $66(38.8)$ & $0.041^{*}$ \\
\hline IFG $(\mathrm{FPG}>100 \leq 126 \mathrm{mg} / \mathrm{dL})$ & $19(16.2)$ & 8 (15.1) & 27 (15.9) & 1.000 \\
\hline $\mathrm{DM}(\mathrm{FPG} \geq 126 \mathrm{mg} / \mathrm{dL})$ & $12(10.3)$ & $4(7.6)$ & $16(9.4)$ & 0.778 \\
\hline WC $>$ cut off ${ }^{*}$ & $22(18.8)$ & $19(35.9)$ & $41(24.1)$ & $0.020^{*}$ \\
\hline Overweight (BMI $\geq 25$ ) & $43(36.8)$ & $17(32.1)$ & $60(35.3)$ & 0.606 \\
\hline Obesity (BMI $\geq 30$ ) & $10(8.6)$ & $7(13.2)$ & $17(10.0)$ & 0.41 \\
\hline MetS & $49(41.9)$ & $15(28.3)$ & $64(37.7)$ & 0.123 \\
\hline
\end{tabular}

*Statistical significant, ${ }^{\dagger} \mathrm{HDL}<40 \mathrm{mg} / \mathrm{dL}$ males and $<50 \mathrm{mg} / \mathrm{dL}$ females, ${ }^{\ddagger} \mathrm{WC}>90 \mathrm{~cm}$ - males and $>80 \mathrm{~cm}$ - females. WC: Waist circumference, TG: Triglycerides, SBP: Systolic blood pressure, DBP: Diastolic blood pressure, HDL: High-density lipoprotein, MetS: MetS: Metabolic syndrome, IFG: Impaired fasting glucose, DM: Diabetes mellitus, BMI: Body mass index 
Table 4: Comparison of prevalence of various disorders in different age groups

\begin{tabular}{|c|c|c|c|c|}
\hline Variables & Age $\leq 40$ years $(\%)$ & Age $>40$ years $(\%)$ & Total (\%) & p value \\
\hline $\mathrm{N}$ & $103(60.6)$ & $67(39.4)$ & $170(100)$ & \\
\hline $\mathrm{SBP} \geq 130 \mathrm{~mm} \mathrm{Hg}$ & $31(30.1)$ & $37(55.2)$ & $68(40.0)$ & $0.001^{*}$ \\
\hline $\mathrm{DBP} \geq 85 \mathrm{~mm} \mathrm{Hg}$ & $30(29.1)$ & $30(44.7)$ & $60(35.3)$ & $0.048^{*}$ \\
\hline $\mathrm{TG} \geq 150 \mathrm{mg} / \mathrm{dl}$ & $37(35.9)$ & $13(19.4)$ & $50(29.4)$ & $0.025^{*}$ \\
\hline HDL $<$ cutoff $^{\dagger}$ & $47(45.6)$ & $19(28.4)$ & $66(38.8)$ & $0.025^{*}$ \\
\hline IFG $(F P G>100 \leq 126 \mathrm{mg} / \mathrm{dL})$ & $9(8.7)$ & $19(28.4)$ & $27(15.9)$ & $0.001^{*}$ \\
\hline $\mathrm{DM}(\mathrm{FPG} \geq 126 \mathrm{mg} / \mathrm{dL})$ & $8(7.8)$ & $8(11.9)$ & $16(9.4)$ & 0.424 \\
\hline Overweight (BMI $\geq 25$ ) & $33(32.0)$ & $27(40.3)$ & $60(35.3)$ & 0.324 \\
\hline Obesity (BMI $\geq 30)$ & $9(8.7)$ & $8(11.9)$ & $17(10.0)$ & 0.602 \\
\hline MetS & $41(39.8)$ & $23(34.3)$ & $64(37.7)$ & 0.519 \\
\hline
\end{tabular}

*Statistical significant ${ }^{\dagger} \mathrm{HDL}<40 \mathrm{mg} / \mathrm{dL}$ males and $<50 \mathrm{mg} / \mathrm{dL}$ females. ${ }^{*} \mathrm{WC}>90 \mathrm{~cm}$ - males and $>80 \mathrm{~cm}$ - females. WC: Waist circumference, TG: Triglycerides, SBP: Systolic blood pressure, DBP: Diastolic blood pressure, HDL: High-density lipoprotein, MetS: Metabolic syndrome, IFG: Impaired fasting glucose, DM: Diabetes mellitus, BMI: Body mass index

Table 5: Percentage of each risk factor contributing to MetS in males and females

\begin{tabular}{llllll}
\hline Gender & TG & HDL & FBS & SBP & WBP \\
\hline $\begin{array}{l}\text { Males } \\
\text { N }(\%)\end{array}$ & $35(29.9)$ & $41(35.0)$ & $28(23.9)$ & $29(24.8)$ & $47(40.2)$ \\
$\begin{array}{l}\text { Females } \\
\text { N }(\%)\end{array}$ & $10(18.9)$ & $15(28.3)$ & $07(13.2)$ & $08(15.1)$ & $10(18.9)$ \\
\hline
\end{tabular}

WC: Waist circumference, TG: Triglycerides, SBP: Systolic blood pressure, DBP: Diastolic blood pressure, HDL: High-density lipoprotein, FBS: Fasting blood sugar, MetS: Metabolic syndrome

The prevalence of MetS in this study showed a higher value for males $(41.88 \%)$ than for the females $(28.30 \%)$ which was statistically insignificant, while a reverse result was observed in earlier studies conducted on the general population $[8,25,27]$. However, our findings were supported by past studies conducted among the doctors where males showed a higher prevalence than the females [15]. Lower prevalence of MetS in this study may be due to the protective effects of endogenous estrogens against the risk factors as more women in the study are aged mostly less than the average age of menopause in India (46.2 years) [29]. Health consciousness among female doctors may be another plausible cause.

Prevalence of MetS is known to increase with age [30]. However, Sawant et al. suggested no significant difference in MetS with age [27]. Few other studies show a higher prevalence of MetS in the younger age group ( $<40$ years) than the older age groups supporting our findings $[6,28]$. Plausible cause of high prevalence in the younger doctors may be due to the high and frequent intake of ready to eat processed food. Lower prevalence among the older group may probably be due to increase consciousness of the health status with increasing age.

Among the other risk factors, high BP and high triglyceridemia were greater in males than females whereas low HDL and high WC were more common in the latter group. Our findings are in support with previous studies [25,31]. A higher prevalence of increased BP and IFG was seen in the elderly group, and higher prevalence of increased TG and decreased HDL was seen in the younger age group [32].

In the males, high DBP was the major contributor to the prevalence of MetS; which was followed by low HDL-C and high TG values. Similarly, low HDL-C and greater WC contributed equally to MetS in females. Greater WC and IFG were the least contributors to MetS in males and females, respectively.

\section{Limitations}

The present study has a few limitations. First, though an adequate sample size has been considered in the study, the study participants are working in a single tertiary care hospital and medical college. Thus, it is difficult to generalize the findings to all the doctors of the community. Second, though few nutritional details of the subjects were collected, correlation with findings could not be done. Third, we did not find out the prevalence of MetS in the general population. Hence, a comparison of findings of the study group with the control group has not been done. Despite the limitations, this study is very few of its kind in India to explore the burden of MetS among the doctors in eastern India. Further probes with the involvement of multi-centers and assessment of other competing risk factors appear necessary to highlight the details of MetS profile among the doctors.

\section{CONCLUSIONS}

In the present study, the young male doctors have a greater prevalence of MetS than the older and the females. MetS is still a significant public health problem in the educated population including those of doctors, especially the younger ones. Active preventive measures such as lifestyle modifications, proper diet, and regular exercise should be undertaken to reduce its prevalence. It is possible that if the doctors themselves practice healthy habits, they would have a greater influence on their patients regarding good health-care practices.

\section{ACKNOWLEDGMENTS}

We are thankful to all the participants of the study. Our thanks to all the paramedical, technical and nontechnical staffs for supporting us in smooth conducting of the study.

\section{AUTHOR'S CONTRIBUTION}

Magna Manjareeka and Suresh Chandra Dash - Planning and Conception of the study. Jayanti Mishra, Soumya Mishra, and Shubhransu Patro - Study design and Acquisition of data. Prakash Kumar Nayak, Soumya Mishra, and Magna Manjareeka - Statistical analysis and interpretation of data. Magna Manjareeka and Prakash Kumar Nayak - Drafting the article or revising it critically for important intellectual content. All Authors - final approval of the version to be submitted.

\section{CONFLICTS OF INTEREST}

Authors do not have any conflicts of interest.

\section{REFERENCES}

1. Bener A, Yousafzai MT, Darwish S, Al-Hamaq AO, Nasralla EA, Abdul-Ghani M, et al. Obesity index that better predict metabolic 
syndrome: Body mass index, waist circumference, waist hip ratio, or waist height ratio. J Obes 2013;2013:269038.

2. Nerkar D, Mukherjee A, Mehta BK, Banerjee S. Metabolic syndrome associated complications. Int J Pharm Pharm Sci 2015;7:22-5.

3. Misra A, Misra R, Wijesuriya M, Banerjee D. The metabolic syndrome in South Asians: Continuing escalation and possible solutions. Indian J Med Res 2007;125:345-54.

4. Hydrie MZ, Shera AS, Fawwad A, Basit A, Hussain A. Prevalence of metabolic syndrome in urban Pakistan (Karachi): Comparison of newly proposed international diabetes federation and modified adult treatment panel III criteria. Metab Syndr Relat Disord 2009;7:119-24.

5. Rampal S, Mahadeva S, Guallar E, Bulgiba A, Mohamed R, Rahmat R, et al. Ethnic differences in the prevalence of metabolic syndrome: Results from a multi-ethnic population-based survey in Malaysia. PLoS One 2012;7:e46365.

6. Pandit K, Goswami S, Ghosh S, Mukhopadhyay P, Chowdhury S. Metabolic syndrome in South Asians. Indian $\mathrm{J}$ Endocr Metab 2012;16:44-55.

7. Das M, Pal S, Ghosh A. Association of metabolic syndrome with obesity measures, metabolic profiles, and intake of dietary fatty acids in people of Asian Indian origin. J Cardiovasc Dis Res 2010;1:130-5.

8. Prasad DS, Kabir Z, Dash AK, Das BC. Prevalence and risk factors for metabolic syndrome in Asian Indians: A community study from urban Eastern India. J Cardiovasc Dis Resv 2012;3:204-11.

9. Hamer M. Psychosocial stress and cardiovascular disease risk: The role of physical activity. Psychosom Med 2012;74:896-903.

10. Petersen CB, Nielsen AJ, Bauman A, Tolstrup JS. Joint association of physical activity in leisure and total sitting time with metabolic syndrome amongst 15,235 Danish adults: A cross-sectional study. Prev Med 2014;69:5-7.

11. Janczura M, Bochenek G, Nowobilski R, Dropinski J, KotulaHorowitz K, Laskowicz B, et al. The relationship of metabolic syndrome with stress, coronary heart disease and pulmonary function - an occupational cohort-based study. PLoS One 2015;10:e0133750.

12. Thayyil J, Jayakrishnan TT, Raja M, Cherumanalil JM. Metabolic syndrome and other cardiovascular risk factors among police officers. N Am J Med Sci 2012;4:630-5.

13. Saini NK, Agrawal S, Bhasin SK, Bhatia MS, Sharma AK. Prevalence of stress among resident doctors working in medical colleges of Delhi. Indian J Public Health 2010;54:219-23.

14. Gupta A, Gupta R, Lal B, Singh AK, Kothari K. Prevalence of coronary risk factors among Indian physicians. J Assoc Physicians India 2001;49:1148-52.

15. Ramachandran A, Snehalatha C, Yamuna A, Murugesan N. High prevalence of cardiometabolic risk factors among young physicians in India. J Assoc Physicians India 2008;56:17-20.

16. Alam MF, Nasreen S, Ullah E, Hussain A. The awareness and prevalence of metabolic syndrome in medical community of Bahawalpur. Oman Med J 2011;26:26-8.

17. Manjareeka M, Mishra J, Nanda S, Mishra S, Padhi RK. Peak expiratory flow rate as a function of anthropometric variables in tribal school children. Int J Physiol 2014;2:4-8.

18. Manjareeka M, Mishra J, Nanda S, Mishra S, Mishra J. Assessment of peak expiratory flow rate in preadolescent children of sub-tribal communities in Odisha. Int J Clin Exp Physiol 2014;1:120-4.

19. Manjareeka M, Nanda S, Mishra J, Mishra S. Correlation between anthropometry and lipid profile in healthy subjects of Eastern India. J Mid Life Health 2015;6:164-8.

20. Ramachandran A, Snehalatha C, Satyavani K, Sivasankari S, Vijay V. Metabolic syndrome in urban Asian Indian adults - a population study using modified ATP III criteria. Diabetes Res Clin Pract 2003;60:199-204

21. Frank E. Physician Health and Patient Care. JAMA 2004;291:637.

22. Oberg EB, Frank E. Physicians' health practices strongly influence patient health practices. J R Coll Physicians Edinb 2009;39:290-1.

23. Ghosh AK, Joshi SR. Physician's health: Time to take care. J Assoc Physicians India 2008;56:13-4

24. Garg PR, Kabita S, Sinha E, Kalla L, Kaur L, Saraswathy KN. The association of non-HDL cholesterol with the presence of metabolic syndrome in North Indian subjects with and without CAD. Ann Hum Biol 2013;40:111-5.

25. Beigh SH, Jain S. Prevalence of metabolic syndrome and gender differences. Bioinformation 2012;8:613-5.

26. Sinha S, Misra P, Kant S, Krishnan A, Nongkynrih B, Vikram NK. Prevalence of metabolic syndrome and its selected determinants among urban adult women in South Delhi, India. J Postgrad Med 2013;89:68-72

27. Sawant A, Mankeshwar R, Shah S, Raghvan R, Dhongde G, Raje H, et al. Prevalence of metabolic syndrome in Urban India. Cholesterol 2011;2011:Article ID: 920983, 7 Pages.

28. Martha S, Ramreddy S, Pantam N. Study of impaired glucose tolerance, dyslipidemia, metabolic syndrome and cardiovascular risk in a south Indian population. J Postgrad Med 2011;57:4-8.

29. Ahuja M. Age of menopause and determinants of menopause age: A PAN India survey by IMS. J Midlife Health 2016;7:126-31.

30. Shalini M, Babu KP, Srinivasa MA, Girish B, Hamsaveena, Mounika K, et al. Metabolic syndrome among urban and rural women population A cross sectional study. J Clin Diag Res 2013;7:1938-40.

31. Gupta R, Deedwania PC, Gupta A, Rastogi S, Panwar RB, Kothari K. Prevalence of metabolic syndrome in an Indian urban population. Int J Cardiol 2004;97:257-61.

32. Pathak KY, Mohanan A, Acharya S, Mandavia D, Jadhav HR. Exploring visceral adiposity index as a predictor of visceral adiposity dysfunction and evaluating its performance in predicting hepatic insulin resistance in Indian Type 2 diabetics. Int J Pharm Pharm Sci 2016;8:297. 